\title{
Resolution of anosmia and urinary incontinence after ablation of supra-ventricular tachycardia
}

\begin{abstract}
Rationale

Supraventricular tachyarrhythmias (SVT) may cause a significant impairment of quality of life. The smell alterations may appear in association with SVT due to side effects of antiarrhythmic drugs ${ }^{1}$ but may as well be a manifestation of another underlying disease..$^{2-4}$

\section{Patient concerns}

In this case report, we describe a case of a 51-year-old female which developed loss of the sense of smell (anosmia) and urine incontinence around the time when she was diagnosed with supraventricular tachycardia. After radiofrequency ablation of atrioventricular nodal re-entry tachycardia (AVNRT) and atrial tachycardia, her ability to smell had recovered and urinary incontinence had improved dramatically. During 18-month follow-up, she reported no complaints of smell anymore. Possible underlying mechanisms are discussed.
\end{abstract}

\section{Lessons}

Our case report describes a patient with a supraventricular tachyarrhythmia who suffered reversible anosmia and urine incontinence which resolved after radiofrequency ablation. Anosmia in our case report had led to a large spectrum of underlying pathologies to consider. The most likely causes of smell disorder in this case were typical aura without headache -a rare type of migraine or somatization disorder. Catheter ablation may have resulted in the reversal of autonomic imbalance causing improvement and later elimination of anosmia.

Keywords: supraventricular arrhythmia, smell, radiofrequency ablation, migraine, Parkinson's disease, anosmia, atenolol, urine incontinence
Volume 12 Issue 4 - 2019

\section{Ella Yahud, Michael Rahkovich, Avishag Laish- Farkash \\ Cardiology Devision, Assuta Ashdod University Medical Center} Israel

Correspondence: Ella Yahud, Cardiology Division, Assuta Ashdod University Hospital, $7 \mathrm{HaRefua}$ Street, Ashdod 7747629, Israel, Tel +972523935075, Fax+972737436226, Email ellaya@assuta.co.il

Received: August 17,2019| Published: August 30, 2019
Abbreviations: SVT, supra-ventricular tachycardia; AV, atrioventricular; AT, atrial tachycardia; CL, cycle length; PD, parkinson's disease; AF, atrial fibrillation

\section{Case presentation}

A 51years old female with a previous history of hypertension, hyperlipidemia and celiac disease presented to our clinic with complaints of palpitations for the last 5years. Her chronic medications were atenolol $12.5 \mathrm{mg}$ once daily and pravastatin $40 \mathrm{mg}$ once daily.

The palpitations were sudden in onset and were terminated by vagal maneuvers. The patient reported no shirt flapping or neck pounding. Her physical examination and echocardiographic exam were normal as well as her blood tests, including thyroid function.

The ECG documented narrow complex short RP tachycardia with ventricular response of $140 \mathrm{bpm}$. The patient noticed that around the time that the symptoms of palpitations appeared for the first time, she had lost the sense of smell and started to suffer from urinary incontinence but didn't search for medical advice. She was assessed in our clinic and decision was made to proceed with electrophysiology study. The study showed dual atrio-ventricular (AV) node physiology. Supra-ventricular tachycardia (SVT) (Figure 1) was induced at a cycle length (CL) of $380 \mathrm{~ms}$ with ventriculo-atrial interval of $35 \mathrm{~ms}$, and entrainment maneuvers supported typical AV node re-entry tachycardia as the working diagnosis. Slow pathway ablation was performed with successful outcome. During isuprel administration and atrial pacing incessant atrial tachycardia (AT) with two different CL of 360 and $320 \mathrm{~ms}$ was provoked (Figure 2).

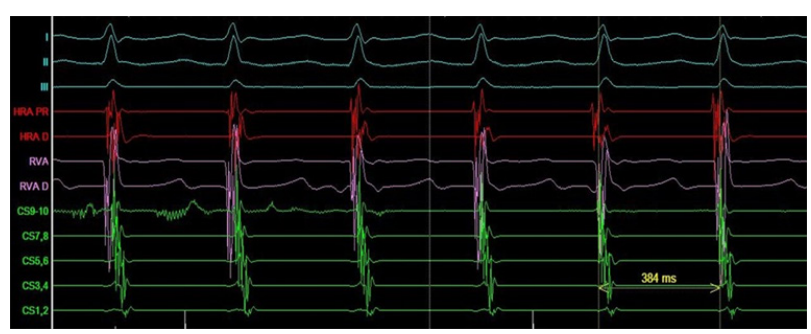

Figure I Typical atrioventricular nodal re-entry tachycardia (AVNRT)

Electrophysiological study: I, II, III- ECG leads; HRA- high right atrium; RVAright ventricular apex; D- distal electrode, PR- proximal electrode; CScoronary sinus

Carto mapping detected 2 foci of AT origin: crista terminalis and right atrial anterior septum (Figure 3). These two foci were ablated and were non-inducible at the end of the procedure. The patient was further discharged with a recommendation to continue treatment with atenolol $12.5 \mathrm{mg}$ once daily. She was then followed-up for 18 months after the ablation in our outpatient clinic. No SVT was detected in repeated continuous Holter monitoring during the follow-up period. 
The patient reported no palpitations; moreover, resolution of anosmia and significant improvement of urinary incontinence occurred immediately after the ablation and ever since.

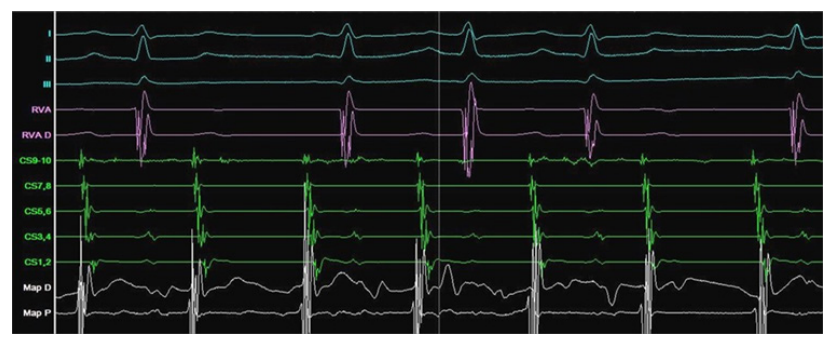

Figure 2 Incessant atrial tachycardia.

Electrophysiological study: I, II, III- ECG leads; RVA- Right ventricular apex; CS- coronary sinus; P- proximal electrodes; D- distal electrodes; Map- mapping catheter
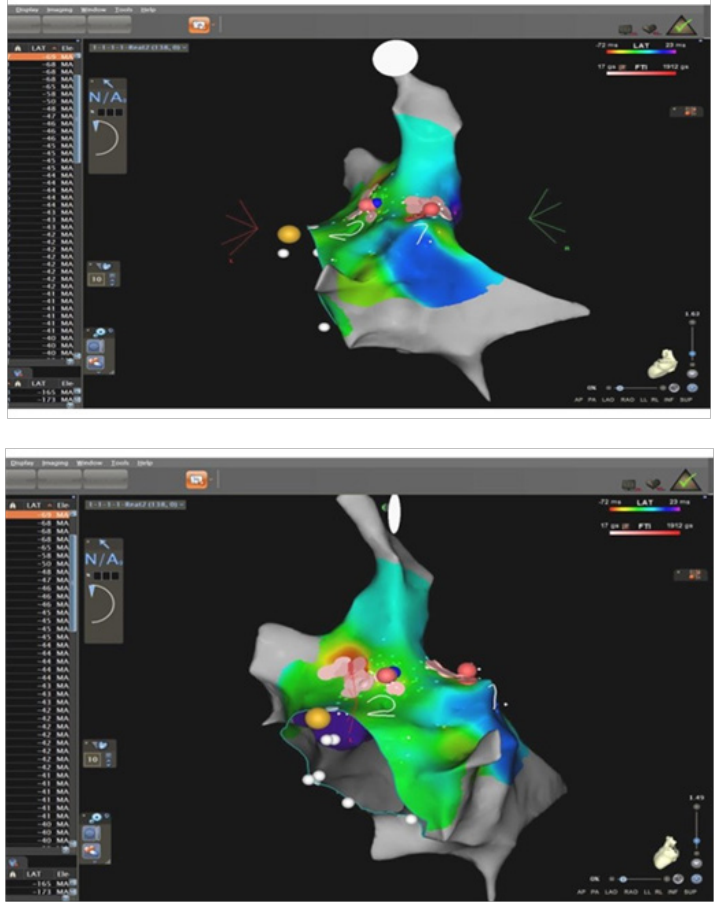

Figure 3 Successful ablation of atrial tachycardia at crista terminalis area and at right anterior septum.

Electroanatomical mapping of right atrium using Carto ${ }^{\circ}$ 3-dimentional mapping system. Red dots show ablation points at Anterior Septum (I) and Crista Terminalis (2) Yellow dot shows His bundle.

\section{Discussion}

We present a unique case of SVT-induced anosmia and resolution of anosmia after radiofrequency ablation of SVT. To the best of our knowledge, this is the first report of such an association. There are several potential causes of anosmia described in the literature. Generally, anosmia is classified as either congenital or acquired.

Congenital anosmia can occur sporadically or be associated with hereditary genetic disorders such as congenital insensitivity to pain, Kallmann syndrome (hypogonadotropic hypogonadism, anosmia, osteoporosis), CHARGE syndrome (coloboma of the eye, central nervous system anomalies, heart defects, atresia of the choanae, retardation of growth and/or development, genital and/or urinary defects, ear anomalies and/or deafness and abnormally shaped ears). ${ }^{3}$ Acquired anosmia may have multiple causes: inflammation in the nasal pathways, ${ }^{4}$ head trauma, olfactory groove tumors, neuro-degenerative disorders (Parkinson's disease, Alzheimer's disease, Lewy body disease), aging, psychiatric disorders, migraine equivalent, somatisation or depression. Anosmia has been linked to several drugs side effects. The most relevant drugs which are used in treating arrhythmias are metoprolol, ${ }^{1}$ digoxin $^{5}$ and amiodarone. ${ }^{6}$

Our patient did not take any of the above mentioned drugs. Moreover, anosmia was resolved after the ablation while atenolol usage was continued; Impaired sense of smell as well as atrial fibrillation ${ }^{7,8}$ may occur in the earliest stages of Parkinson's disease (PD) and may precede motor symptoms by several years. The potential mechanism is an impairment of nerve cell growth in the region of olfactory bulb due to dopamine depletion which occurs in PD. ${ }^{2}$ The relative young age, the association with SVT timing, and the absence of motor symptoms years after appearance of anosmia make the diagnosis of PD unlikely in our patient; another possible cause of anosmia in this patient is a migraine aura. Aura can appear at some point in $38 \%$ of patients with migraine headaches. Moreover, typical aura without headache occurs in $4 \%$ of patients with diagnosis of migraine. Aura may present as olfaction-related symptoms such as olfactory hallucinations $(6.2 \%)$, phantosmia (4.4\%), cacosmia/euosmia $(2.6 \%) .{ }^{9}$ There is evidence to support a beneficial effect of atrial fibrillation (AF) ablation on duration and intensity of pain in migraine. ${ }^{10}$

Autonomic dysfunction seems to be involved in the pathophysiology of both migraine and SVT. Concomitant sympathetic hyperfunction and parasympathetic hypofunction was reported to be present in patients with migraine. ${ }^{11}$ Catheter ablation of AF decreases an efferent sympathetic nerve activity and stimulates postganglionic efferent parasympathetic fibers. ${ }^{12}$ Therefore, catheter ablation might play role in the the reversal of autonomic imbalance causing elimination or improvement of clinical symptoms of migraine in AF patients. ${ }^{13}$ Its effect during SVT ablation may be similar but was not proved yet;

It is known that olfactory symptoms can be one of obscure manifestations of somatization and depressive disorders. ${ }^{14}$ It seems reasonable that improvement in quality of life and mood after SVT ablation may underlie the improvement of the olfactory symptoms in our patient.

As described previously, ${ }^{15}$ paroxysmal SVT might cause polyuria, increasing the urinary flow by twice at the end of SVT episode. The underlying mechanisms include increased release of atrial natriuretic peptide related to left atrial strain, increased anti diuretic hormone plasma level and increased levels of prostaglandin E2 stimulated by anti-diuretic hormone, similar to the mechanisms that underlie polyuria in AF patients. ${ }^{16}$ Successful ablation of SVT may therefore lead to significant improvement in polyuria and urinary incontinence, as described in our patient.

\section{Conclusions}

We report a unique case of resolution of anosmia and urinary incontinence associated with successful SVT ablation. Further studies investigating the underlying mechanisms are needed.

\section{Acknowledgments}

None. 


\section{Conflicts of interest}

The authors declare that there are no conflicts of interest

\section{References}

1. Che X, Li Y, Fang Y, et al. Antiarrhythmic drug-induced smell and taste disturbances. A case report and literature review. Medicine(Baltimore). 2018;97(29):e11112.

2. Wiley-Blackwell. Impaired Sense of Smell May Be Early Indicator of Parkinson's Disease. Science Daily. 2008.

3. Karstensen HG, Tommerup N. Isolated and syndromic forms of congenital anosmia. Clin Genet. 2012;81(3):210-215

4. Knight A. Anosmia. The Lancet. 1988;332(8609):512.

5. Ishimaru T, Yokogawa H. Olfactory and gustatory disturbances caused by digitalism: a case report. Auris Nasus Larynx. 2006;33(4):465-469.

6. Maruyama T, Yasuda S, Odashiro K, et al. Anosmia induced by amiodarone. Am J Med. 2007;120(11):e9.

7. Hong CT, Chan L, Wu D, et al. Association between Parkinson's disease and atrial fibrillation: a population-based study. Front Neurol. 2019;10:22.

8. Fullard ME, Mrley JF, Duda JE. Olfactory dysfunction as an early biomarker in Parkinson's disease. Neurosci Bull. 2017;33(5):515-525.

9. Fornazieri MA, Neto AR, de Rezende Pinna F, et al. Olfactory symptoms reported by migraines with and without auras. Headache. 2016;56(10):1608-1616.
10. Mohanty S, Mohanty P, Rutledge JN, et al. Effect of catheter ablation and periprocedual anticoagulation regimen on the clinical course of migraine in atrial fibrillation patients with or without pre-existent migraine: results from a prospective study. Circ Arrhythm Electrophysiol. 2015;8(2):279287.

11. Appel S, Kuritzky A, Zahavi I, et al. Evidence for instability of the autonomic nervous system in patients with migraine headache. Headache. 1992;32(1):10-17.

12. Chen PS, Tan AY. Autonomic nerve activity and atrial fibrillation. Heart Rhythm. 2007;3(3 Suppl):S61-S64.

13. Mohanty S, Mohanty P, Rutledge JN et al. Effects of catheter ablation and periprocedual anticoagulation regimen on the clinical course of migraine in atrial fibrillation patients with or without pre-existent migraine. Circ Arrhythm Electrophysiol. 2015;8(2):279-287.

14. Alam ES, Musselman DL, Chyou D et al. Somatization, depression and anxiety disorders in a rhinology practice. Am J Rhinol Allergy. 2019;5:1945892419841317.

15. Fujii T, Kojima S, Imanishi M, et al. Different mechanisms of polyuria and natriuresis associated with paroxysmal supraventricular tachycardia. Am J Cardiol. 1991;68(4):343-348.

16. Roy D, Paillard F, Cassidy D, et al. Atrial natriuretic factor during atrial fibrillation and supraventricular tachycardia. JACC. 1987; 9(3):509-514. 